\title{
Study of Gas Permeation Through Thin ta-C:H Films
}

\author{
J.M. LACKNER ${ }^{a}$, W. WALDHAUSER ${ }^{a, *}$, M. KAHN $^{b}$ \\ ${ }^{a}$ Joanneum Research Forschungsgesellschaft mbH, Institute for Surface \\ Technologies and Photoniks, 8712 Niklasdorf, Austria \\ ${ }^{b}$ Infineon Technologies Austria AG, 9500 Villach, Austria
}

\begin{abstract}
Protective ultra-thin barrier films gather increasing economic interest for controlling permeation and diffusion from the biological surrounding in implanted sensor and electronic devices in future medicine. Thus, the aim of this work was the investigation of the film thickness influence on the gas permeation barrier of ultra-thin, cytocompatible tetrahedral amorphous carbon $(\mathrm{ta}-\mathrm{C}: \mathrm{H})$ films on polyimide (PI) foils. Plasma-activated chemical vapor deposition (direct deposition from an ion source) was applied to deposit these diamond-like carbon films. The results indicate high barrier to hydrogen gas permeation by all film thicknesses $\left(<0.2 \% \mathrm{H}_{2}\right.$ permeation compared to uncoated PI). While the thickness of the ta-C:H layers has minor influence, the number of layers, realized by one- or double-side deposition strongly impacts the barrier effect. Finally, tests under tensile stresses showed minor impact in the elasto-plastic deformation regime, but the expected strong increase of gas permeation after exceeding the tensile strength and film fracture.
\end{abstract}

DOI: 10.12693/APhysPolA.127.1236

PACS: $73.50 .-\mathrm{h}$

\section{Introduction}

Finding materials with both high biocompatibility (cytocompatibility, hemocompatibility, etc.) and low or controllable gas permeation is a great challenge for using microelectronics and microfluidics in in vivo sensors. Microelectronic devices are generally based on a large number of materials for electronics and printed circuit boards, including ceramics and polymers for chip and resistor housings, metal and semiconductors for the chip itself, metals (Al, Ni-Au, $\mathrm{Pd}-\mathrm{Au}, \mathrm{Cu}, \mathrm{Pd}-\mathrm{Cu}$, etc.) for circuit paths, fiber reinforced epoxy binders, polyimide and polyurethane foils for dielectrics and various glues. Only a few of them are biocompatible, as shown in tests with hippocampus cells by Mazzuferi et al. [1]. Thus, dense packaging for any in vivo use is essential to prevent permeation/diffusion of body fluids into the electronic components. This saves the electronics from biological fluids as well as the tissue from any (corrosion) contaminants from the electronics.

Packaging by metal or polymer housings is state-ofthe-art, but limited in miniaturization. Coatings are alternatives to such robust housings, but they must prove their applicability both for biocompatibility and for long-term permeation and corrosion protection. In recent years, polymers like polydimethylsiloxane, parylene$\mathrm{C}$ and polymethyl methacrylate have been applied on silicon based devices due to easy manufacturing, sufficiently high mechanical properties and bio-inertness. However, their barrier properties are limited, as shown for parylene-C in contact with blood [2]. Polyelectrolyte

\footnotetext{
* corresponding author; e-mail: wolf gang. waldhauser@joanneum. at
}

layers are generally robust, but possess no long-term stability [3, 4]. Plasmapolymer layers, deposited from hydrocarbon, silicon and titanium containing organic compounds or monomers like tetraglyme, are generally free of pin-holes and possess good barrier properties. Their adhesion to microelectronic substrate materials is high [5]. Inorganic thin films based on $\mathrm{SiO}_{2}$ and $\mathrm{SiN}$ are frequently used as permeation protective coatings for microelectronic and microfluidic devices due to their bioinert behavior, missing cytotoxicity and high barrier effect [6]. Other well-known vacuum coating materials, deposited by physical and chemical vapor deposition (PVD, CVD) are platinum, gold, titanium oxide and other ceramics like aluminum oxide and oxynitride [7]. Further improvements in gas barrier are provided by multilayered film structures based on inorganic and organic layers [8]. Ultra-thin and dense films can be grown by atomic layer deposition (e.g. $\mathrm{Al}_{2} \mathrm{O}_{3}, \mathrm{SiC}, \mathrm{TiO}_{2}[9]$ ). However, they have application limitations for low-temperature resistant substrates like polymers due to the required high temperatures for precursor dissociation. Similar problem exists for ultra nanocrystalline diamond thin films, which have a high gas permeation barrier and are not cytotoxic, but require $400-800{ }^{\circ} \mathrm{C}$ temperature for phase formation during deposition. In contrast, diamond-like carbon based materials (DLC) require only lower temperatures for film growth by various technologies (e.g. magnetron sputtering, filtered arc deposition, pulsed laser deposition, plasma-activated chemical vapor deposition (PACVD), etc.). Such DLC coatings are generally amorphous and are characterized by a mixture of $\mathrm{sp}^{2}$ and $\mathrm{sp}^{3}$ hybridized carbon atoms and, mostly, hydrogen $(\mathrm{a}-\mathrm{C}: \mathrm{H})$. The cytotoxicity of DLC materials is generally very low with no in vitro effects on mouse macrophages, human fibroblasts, monocytes, osteoblasts [10] even under in vivo conditions in subcutaneous, bone and muscle tissue in guinea pig, sheep and rat models). 
Minimizing defects and achieving high density DLC films, which is critical for high gas barrier [11] requires the application of deposition techniques with low rates as well as gaseous carbon sources and low gas pressures. Direct deposition from high energetic, but nearly neutralized plasma of ion sources is one of these techniques, where highly pure gaseous precursors (e.g. methane, ethyne) are introduced into high vacuum and ionized by the discharge in an ion source on either direct or indirect path by excited inert gas atoms. While industrially widely-used end-Hall sources provide dense plasma with high particle flux, but low energy, the comparatively new, easily linear-upscaleable anode layer ion source (ALS) technology produces low flux, high energetic plasma [12]. We investigated hydrogenated tetrahedral amorphous carbon films (ta-C:H), which contain mainly $\mathrm{sp}^{3}$ hyridized carbon atoms, from ALS direct deposition in the past on their mechanical [12] as well as cytotoxic behavior [13] and found nanohardness values between 24 and $36 \mathrm{GPa}$ as well as reduced elastic modulus between 140 and $180 \mathrm{GPa}$, depending on the deposition parameters (especially the discharge voltage), as well as no cytotoxic effects in vitro. In contrast, these ta-C:H films boost the fibroblast cell growth in comparison to reference glass substrates.

The goal of this work is the study of the film thickness influence on the gas permeation as well as the description of the impact of tensile stresses on gas permeation.

\section{Experimental}

\subsection{Polymer substrates and thin film deposition}

The deposition of the coatings was performed by vacuum coating equipment at Joanneum Research (for a detailed description see [14]). Before deposition, we cleaned the polyimide (PI) substrates $(25 \mu \mathrm{m}$ thickness, DuPont, Vespel $\left.{ }^{\circledR}\right)$ with isopropanol and dried them in vacuum. After removing any dust particle in nitrogen flow and mounting the substrates parallel to the surface of the deposition source in $\sim 100 \mathrm{~mm}$ distance, we pumped the vacuum chamber down to at least $4 \times 10^{-3} \mathrm{~Pa}$. The ta-C:H thin films were deposited by high-energetic direct deposition at room temperature from ALS plasma (ALS340, Veeco Process Equipment Inc., Fort Collins, $\mathrm{CO}$, USA) from ethyne precursor $\left(\mathrm{C}_{2} \mathrm{H}_{2}, 99.8 \%\right.$ purity, Linde-Gas, Stadl-Paura, Austria). During the flow through the ALS, ionization in the magnetic field occurs. However, the cathode design enable a rather fully neutralization of the ions and the formation of an $\mathrm{C}_{2} \mathrm{H}_{2}$ plasma with a high content of atoms with high kinetic energy [15]. Formed films have a nanohardness of 25.5 GPa and a reduced elastic modulus of $152 \mathrm{GPa}$, both measured by nanoindentation with a Berkovich indenter with a maximum penetration depth of $10 \%$ of the film thickness [16]. The chemical composition of the films, based on Raman investigations, reveals an intensity ratio $I_{D} / I_{G}$ of the fitted $D$ and $G$ band in the carbon spectra of 0.59 and a full-width at half-maximum of the $G$ band of $183 \mathrm{~cm}^{-1}$ [12].

\subsection{Thin film characterization}

The hydrogen permeation was measured based on the carrier gas method (DIN 53380-3) for flat polymers. The experimental setup is based on two evacuated chambers, which are separated and sealed by the (coated) polymer foil specimen. In one of the chambers, a defined hydrogen partial pressure is afterwards introduced and the change of pressure in the second chamber by the gas permeation through the specimen is measured. The detection limit of this method is $0.01 \mathrm{~cm}^{3} \mathrm{~m}^{-2} \mathrm{~d}^{-1}$, but reliable, reproducible measurements require $<0.1 \mathrm{~cm}^{3} \mathrm{~m}^{-2} \mathrm{~d}^{-1}$ gas permeation rate due to influences of the specimen material, surface and thickness. Dry hydrogen with 99.5\% purity was applied as test gas with 1 bar pressure $(101325 \mathrm{~Pa})$ in one chamber, similar pressure of dry air was continuously purged through the second chamber. Hydrogen partial pressure measurements from this purged air occurred by a hydrogen leak detector (H2000 Plus, Adixen, Annecy, France) with a detection range of $0.5-100 \mathrm{ppm}_{2}$ in a measurement device owned by Mecadi (Bexbach, Germany). Temperatures of 22, 50 and $100{ }^{\circ} \mathrm{C}\left( \pm 2{ }^{\circ} \mathrm{C}\right)$ were selected for detecting the temperature influence. The device allowed a tensile straining of the foils too: Therefore, the specimen is clamped and elongated mechanically.

\section{Results and discussion}

Figure 1 shows the measured hydrogen gas permeation rates in dependency on the film thickness on single and double side coated PI substrates in dependency on the film thickness and the test temperature. As expected, the increase of temperature (e.g. from 22 to $100{ }^{\circ} \mathrm{C}$ ) increases the permeation of hydrogen by a factor of $8-12$. Compared to the uncoated PI, the ta$\mathrm{C}: \mathrm{H}$ thin film, deposited only on one side, decreases rather independently on the film thickness the hydrogen permeation by a factor of $\sim 25$. Thin films on both substrate sides enable a further decrease in hydrogen transportation through the foil by a factor of $\sim 10$. The smallest hydrogen permeation rate was found for the $25 \mathrm{~nm}$ ta-C:H films on both sides of the PI with about $2.5 \mathrm{~cm}^{3}(\mathrm{STP}) \mathrm{d}^{-1} \mathrm{bar}^{-1}$, which leads related to the measured film area and film thickness to about $4 \times 10^{-4} \mathrm{~cm}^{3}$ (STP) $\mathrm{mm} \mathrm{m}^{-2} \mathrm{~d}^{-1} \mathrm{bar}^{-1}$. This calculated hydrogen permeability value is very low in comparison to state-of-the-art materials: Polymer materials, normalized to their thickness, present permeabilities between 10 (polyether ether ketone, polyphenylene sulphide) and several $100 \mathrm{~cm}^{3}$ (STP) $\mathrm{mm} \mathrm{m}^{-2} \mathrm{~d}^{-1} \mathrm{bar}^{-1}$ (polyethylene, polytetrafluoroethylene, perfluoroalkoxy alkane). Deposited thin films, especially aluminum, results in permeabilities of $0.1 \mathrm{~cm}^{3}(\mathrm{STP}) \mathrm{mm} \mathrm{m}^{-2} \mathrm{~d}^{-1} \mathrm{bar}^{-1}$ and below [17].

The low dependency of hydrogen permeation on film thickness and high impact by single or double side coating leads to a defect controlled mechanism of hydrogen permeation in the thin ta- $\mathrm{C}: \mathrm{H}$ films. Micro- and 


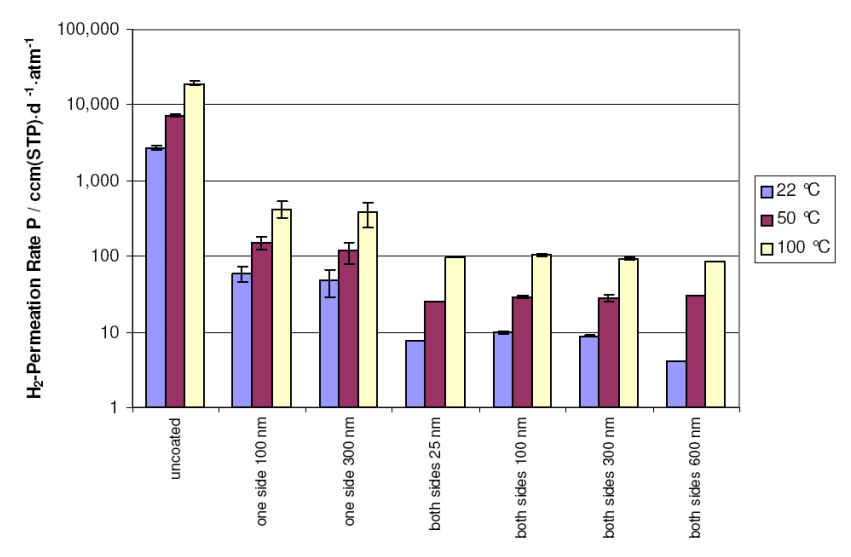

Fig. 1. Hydrogen permeation rate of single and double side ta-C:H coated PI foils with various film thicknesses in comparison to the uncoated PI and in dependency of the test temperature.

nano-pores are generally responsible for such defect controlled mechanism on highly smooth materials like the applied PI substrates: Micro-pores are visible by light microscopy and have their origin either in substrate microstructure (like fillers or reinforcement particles and fibers, phase structure by the mixture of semicrystalline and amorphous phases etc.), the substrate surface (contaminations, scratches, dust particles, etc.) as well as the thin film itself (delamination of the film or droplets and particles incorporated in the film during deposition). Nano-pores are due to the intrinsic film structure (e.g. intercolumnar diffusion in crystalline films, low packing density in amorphous films) [18]. Due a general much higher density of nano-pores over the whole coated area in comparison to micro-pores, the permeation is mainly dependent on gas transport along the nano-pores [11]. This can also be stated for the ta-C:H films on PI due to very smooth and scratch free PI with amorphous structure and without any filler, good chemical and plasma cleaning before film deposition. Film surfaces are quite free of any microscopically visible particle. Also the calculated activation energy for permeation, which is for single side coated PI between 24 and $25 \mathrm{~kJ} \mathrm{~mol}^{-1}$ and distinctly different to the double side coated PI with about $32-34 \mathrm{~kJ} \mathrm{~mol}^{-1}$ (uncoated PI: $23 \mathrm{~kJ} \mathrm{~mol}^{-1}$. Such calculation is possible due to Arrhenius temperature dependency of permeation and the availability of measurements at different temperatures.

To estimate the influence of deformation on the permeability, we performed such an experiment with a double-side ta-C:H coated PI with $25 \mathrm{~nm}$ film thickness. The permeation rate increases from $\sim 0.02 \mathrm{~cm}^{3}$ (STP) $\mathrm{mm} \mathrm{m}^{-2} \mathrm{~d}^{-1} \mathrm{bar}^{-1}$ at the non-stressed condition to $\sim 0.025 \quad \mathrm{~cm}^{3}(\mathrm{STP}) \mathrm{mm} \mathrm{m}^{-2} \mathrm{~d}^{-1} \mathrm{bar}^{-1}$ at $0.5 \%$ relative elongation and to $\sim 0.12 \mathrm{~cm}^{3}$ (STP) $\mathrm{mm} \mathrm{m}^{-2} \mathrm{~d}^{-1} \mathrm{bar}^{-1}$ at $1 \%$ elongation. This permeation rates are similar to ceramic-like silicon oxide coatings [17] and about 8 times higher to highly plastically deformable thin aluminum films.
Because of the very different elastic modulus of thin films and the PI substrates, differing by the factor $\sim 80$, small elastic deformations of the substrate leads to high stresses in the thin films. Reasonably, the ta-C:H film cracks along lines perpendicular to the straining direction (compare to finding in [19]), which was found in own tensile straining on polyurethanes too [20]. A similar effect of thin film fracture by drastically higher stress level influences the increase of permeability at elevated temperatures, as found in Fig. 1: The thermal expansion of polyimide is about 10 times higher than that of ta-C:H, which results in biaxial elastoplastic deformation of the films (including wrinkling phenomena and wave formation of the compound) and fracture. However, if the deformation of the PI is only elastic, unloading or temperature decrease will fully close all cracks, impeding their investigation. The investigation of the influence of this crack closing on the gas permeation will be the topic of the ongoing research.

\section{Conclusions}

This work presented good barrier effect against hydrogen permeation by ta-C:H films, which is mainly influenced by the number of layers (single or double side coated PI substrates). Temperature and mechanical stress have similar influence due to the highly different elastic modulus of the polymer substrate and carbon thin film, resulting in increasing hydrogen permeation. Relating the gas permeation on the film thickness, extremely low values are evident in comparison to other thin films and polymers.

This work gives the basis for ongoing development of multilayered structures by introduction of soft, flexible layers in between of hard, permeation barrier layers.

\section{Acknowledgments}

The financial support of this work by the Austrian Federal Ministry of Traffic, Innovation and Technology, Austrian and the Austrian Research Promotion Agency within the frame of the Austrian research programme "Intelligent Production", and the transnational MNTERA.NET programme with additional support of the European Union is highly acknowledged. Further thanks for financial support are granted to the Federal Country of Styria (Austria). The authors want to thank Dr. Andreas Konrad from Mecadi GmbH, Bexbach (Germany) for permeation measurements and Bettina Grossschaedl from Joanneum Research for coating deposition.

\section{References}

[1] M. Mazzuferi, R. Bovolenta, M. Bocchi, T. Braun, J. Bauer, E. Jung, R. Gambari, Biomaterials 31, 1045 (2010)

[2] D. Feili, M. Schuettler, T. Doerge, S. Kammer, T. Stieglitz, Sensors Actuators A Phys. 120, 101 (2005).

[3] H. Makamba, J.H. Kim, K. Lim, N. Park, J.H. Hahn, Electrophoresis 24, 3607 (2003). 
[4] W.C. Sung, C.C. Chang, H. Makamba, S.H. Chen, Anal. Chem. 80, 1529 (2008).

[5] M. Walther, M. Heming, M. Spallek, Surf. Coatings Technol. 80, 200 (1996).

[6] S.D. Senturia, Microsystem Design, Springer Science+Business Media, LLC: New York, NY, USA, 2005.

[7] C. Charton, N. Schiller, M. Fahland, A. Holländer, A. Wedel, K. Noller, Thin Solid Films 502, 99 (2006).

[8] A. Hogg, H. Keppner, T. Aellen, J. Burger, Ultra-Thin Multi-Layer Protection, US Patent 2011/0039050 A1, 2011.

[9] N.D. Hoivik, J.W. Elam, R.J. Linderman, V.M. Bright, S.M. George, Y.C. Lee, Sensors Actuators A Phys. 103, 100 (2003).

[10] S. Linder, W. Pinkowski, M. Aepfelbacher, Biomaterials 23, 767 (2002).

[11] Y. Letterier, Progr. Mater. Sci. 48, 1 (2003).

[12] M. Kahn, M. Cekada, T. Schöberl, R. Berghauser, C. Mitterer, C. Bauer, W. Waldhauser, E. Brandstätter, Thin Solid Films 517, 6502 (2009).

[13] J.M. Lackner, C. Meindl, C. Wolf, A. Fian, C. Kittinger, M. Kot, L. Major, C. Czibula, C. Teichert, W. Waldhauser, A.-M. Weinberg, E. Fröhlich, Coatings 3, 268 (2013).
[14] J.M. Lackner, Habilitation Thesis, Polish Academy of Sciences, Institute of Metallurgy and Materials Science, Krakow (Poland), Orekop 2005.

[15] J.M. Lackner, W. Waldhauser, M. Schwarz, L. Mahoney, L. Major, B. Major, Vacuum 83, 302 (2008).

[16] M. Kahn, M. Cekada, R. Berghauser, W. Waldhauser, C. Bauer, C. Mitterer, E. Brandstätter, Diam. Rel. Mater. 17, 1647 (2008).

[17] A. Konrad, B. Pannwitt, R. Hempelmann, F. Arena, W. Waldhauser, L. Strack, W. Oelerich, M. Block, Permeation and Leak Testing of Composites for Tank Systems under Tensile Stress, Presentation at Tank.Tech 2009 Meeting, 10./11.11.2009.

[18] B.M. Henry, A.P. Roberts, C.R.M. Grovenor, A.P. Sutton, G.A.D. Briggs, Y. Proc. $41^{\text {st }}$ Society of Vacuum Coaters Annual Conference, Boston, April 18-23, SVC, 434 (1998).

[19] D.R. Wheeler, H. Osaki, ACS Symposium Series 440 , 500 (1990).

[20] J.M. Lackner, W. Waldhauser, R. Major, L. Major, P. Hartmann, Surf. Coatings Technol. 215, 192 (2013). 